\title{
Changing dynamics: Time-varying autoregressive models using generalized additive modeling
}

\author{
Laura F. Bringmann \\ Ellen L. Hamaker \\ Daniel E. Vigo \\ André Aubert \\ Denny Borsboom \\ Francis Tuerlinckx
}

\section{Supplementary material: Details of the simulation setup}

The description of the simulation study is divided into three steps: (1) simulation conditions, (2) generating the simulation data and (3) model estimation and evaluation. An overview of these three steps of the simulation is given in Figure 1. The programming language $R$ was used for all statistical simulations and analyses.

Step 1: Simulation conditions. We varied three factors: 1) the generating function of $\beta_{0, t}$ and $\beta_{1, t}$ (invariant, linear, cosine, random walk and stepwise); 2) the maximum absolute value of the timevarying parameters (low or high); and 3) the sample size (30,60, 100, 200, 400, 1000). This resulted in $60(5 \times 2 \times 6)$ different conditions. A total of $R=1000$ replications of each condition were simulated. We elaborate on the factors below.

1. Parameter generating functions. The intercept $\left(\beta_{0, t}\right)$ and autoregressive parameter $\left(\beta_{1, t}\right)$ of the TV-AR model were generated with five different types of functions, three of them gradually changing and two non-gradually changing. The function generating the attractor $\left(\mu_{t}\right)$ was indirectly calculated afterwards, see Figure 1. 
The first of the gradually changing functions is a time invariant function, meaning that the $\beta_{0, t}$ and $\beta_{1, t}$ do not change over time and could therefore also be modeled with a standard AR. The second is a linear function. In this case $\beta_{0, t}$ and $\beta_{1, t}$ increase over time. The third of the gradually changing functions is a cosine function, where $\beta_{0, t}$ and $\beta_{1, t}$ first increase, then decrease and in the end increase again. The fourth and fifth functions are non-gradually changing, and thus violate the assumption of gradual change of the TV-AR model. The fourth function is a random walk function, in which $\beta_{0, t}$ and $\beta_{1, t}$ are generated in such a way that they show random and fast change that can also result in an increase or decrease in the function over a period of time. The fifth function is a stepwise function, meaning that $\beta_{0, t}$ and $\beta_{1, t}$ have for a certain period of time a constant value, which then changes abruptly to a higher value.

2. Low and high maximum values. Besides the different generating functions, we also compared low and high value settings for the maximum absolute values possible for the time-varying parameters. The maximum absolute values for the low condition for $\beta_{0, t}$ (the intercept) were 1 and for the high condition 1.5. Thus, for example, the peak values for the cosine function were 1 and -1 in the low condition (and 1.5 and -1.5 in the high condition). The maximum absolute value for the low and high condition for $\beta_{1, t}$ (the autoregressive parameter) was set to 0.2 and 0.5 , respectively (based on values typically found in psychological studies, see e.g., Rovine \& Walls, 2006). Whereas the invariant, linear, cosine and stepwise function are by definition bounded, a random walk is not, so in order to have a bounded random walk with the above mentioned maximum absolute values we used an adapted version of the formula $\rho a_{t} / \max _{0 \leq j \leq t}\left|a_{j}\right|$, based on Giraitis, Kapetanios, and Yates (2014). This formula guarantees that the random walk will be bounded between the pre-specified $-\rho$ and $\rho$. In this formula, $a$ is defined as follows: $a_{t}-a_{t-1}=\eta_{t}$. Here, the difference between $a_{t}$ and $a_{t-1}$ equals $\eta_{t}$, a random number drawn from an independent identically normal distribution. At every time point $\rho$ is multiplied with $a_{t}$ and then divided by the maximum absolute value of $a$ up to current time point $t$.

3. Sample size. Furthermore, sample sizes (the number of time points, $n$ ) were chosen to be comparable to those possible in psychological research: 30, 60, 100, 200, 400 and 1000. This will shed light on the amount of time points needed in order for the TV-AR to give a reliable recovery of the "true" underlying model. ${ }^{1}$

\footnotetext{
${ }^{1}$ As pointed out by an anonymous reviewer, the local range of change of the cosine function is dependent upon sample
} 
Step 2: Generating the simulation data. To generate the simulation data, we used the TV-AR formula introduced in section 3: $y_{t}=\beta_{0, t}+\beta_{1, t} y_{t-1}+\varepsilon_{t}$ (see also step 2 in Figure 1). The time-varying intercept $\beta_{0, t}$ and the time-varying autoregressive parameter $\beta_{1, t}$ can be generated after the parameter generating function, the maximum absolute value of the parameters and the sample size have been set. The residuals $\varepsilon_{t}$ are a white noise process. This is simulated by drawing $n$ times (with $n$ being the number of time points) randomly from a standard normal distribution $\mathcal{N}(0,1)$. Since the model is an autoregressive model with a lagged variable, we had to pre-specify the zeroth observation $\left(y_{0}\right)$, which we drew from a stationary marginal normal distribution:

$$
\mathcal{N}\left(\frac{\beta_{0}}{1-\beta_{1}}, \frac{\sigma_{\varepsilon}^{2}}{1-\beta_{1, t}^{2}}\right)
$$

Marginal means here that the time point is not conditioned on the previous time point (see also the Appendix, the $R$-code). Now all further time points of $y_{t}$ can be simulated. Note that the generated time series, as can been seen in Figure 1, follows the trajectory of the attractor $\left(\mu_{t}\right)$.

Step 3: Estimation and evaluation. We used seven different settings for estimating $\beta_{0, t}$ and $\beta_{1, t}^{2}: 1$ ) a TV-AR model using the default setting (a thin plate regression spline basis using 10 basis functions); 2) a TV-AR model with only a time-varying intercept and a time-invariant autoregressive parameter using the default settings; 3) a TV-AR model with only a time-varying autoregressive parameter using the default settings; 4) a standard time-invariant AR model; and 5) a thin plate regression spline basis using 30 basis functions.

Although 10 basis functions is the standard setting in the mgcv package, this might not always be enough to capture the wiggliness of a function, especially when a function takes a lot of turns, as is the case with for example the random walk function. Therefore, it is interesting to check whether an increase in basis functions leads to better estimations. However, increasing the number of basis functions requires that there is a large amount of time points. In this simulation, at least 400 time points were needed for increasing the number of basis functions to 30. Thus, we could only compare the difference between 10 and 30 basis functions for the sample sizes $n=400$ and $n=1000$ time points. Note that sometimes with already 100 time points it is possible to estimate a TV-AR model with 30 basis functions. However, from 400 time points on, the TV-AR model with 30 basis functions could be fitted for all 1000 replications, whereas with less than 400 time points this was not always the case.

size: there is a smaller rate of change for larger sample sizes.

${ }^{2}$ The attractor $\mu_{t}$ was again indirectly derived from the results of $\beta_{0, t}$ and $\beta_{1, t}$ (see Figure 1 step 3). 
To evaluate the global performance of the TV-AR model, we used the log of the median of the mean squared errors (MSEs) of the $R=1000$ replications per condition. The MSE for a single timevarying parameter is defined as $\frac{1}{n} \sum_{t=1}^{n}\left(\hat{\theta}_{t}-\theta_{t}\right)^{2}$, in which $\hat{\theta}_{t}$ stands for the estimated value at time point $t$ and $\theta_{t}$ for the true value at time point $t$. Any of the parameters $\beta_{0, t}, \beta_{1, t}$ or $\mu_{t}$ can take the role of $\theta_{t}$ and $n$ stands for the number of time points. In addition, a coverage probability was calculated, which is the proportion of time that the true value is captured by the constructed CIs. ${ }^{3}$

An example is given in step 3 of Figure 1. All parameters have been estimated with our TV-AR model after the data were generated. The estimated values $\hat{\theta}_{t}$ and the true values $\theta_{t}$ of the parameters are represented as the middle solid black and red lines respectively. In this figure, the black and red solid line are close to each other, meaning that the model estimates had a low MSE value and the true underlying function(s) could be estimated well. The estimated CIs corresponding to the dashed lines show that almost everywhere the true values of the function are within these intervals, meaning that also the coverage probability was very high and the TV-AR model could estimate the true underlying model well.

Finally, we evaluated whether we could discriminate between a time-varying and time-invariant model (models 1, 2, 3 and 4 of step 3). We used the AIC, BIC and GCV to select the best fitting model for every replication. Next we calculated how often the correct model was selected by these fit indices. For example, for the cosine generating function condition we calculated how often the BIC correctly indicated that model 1, 2 or 3 (time-varying models) was the best fitting model versus model 4 (time-invariant model). In addition, the type I and type II errors were calculated. Finally, for both the intercept and autoregressive parameter, if applicable, the effective degrees of freedom (edf) and $p$-values were extracted.

\footnotetext{
${ }^{3}$ Although the CIs are given as output for the intercept and the autoregressive parameter, this is not the case for the attractor, since this time-varying parameter is only estimated indirectly. Therefore, for the smooth function of the attractor we calculated the CIs independently following the same procedures as in the mgcv package (see also the R-code).
} 

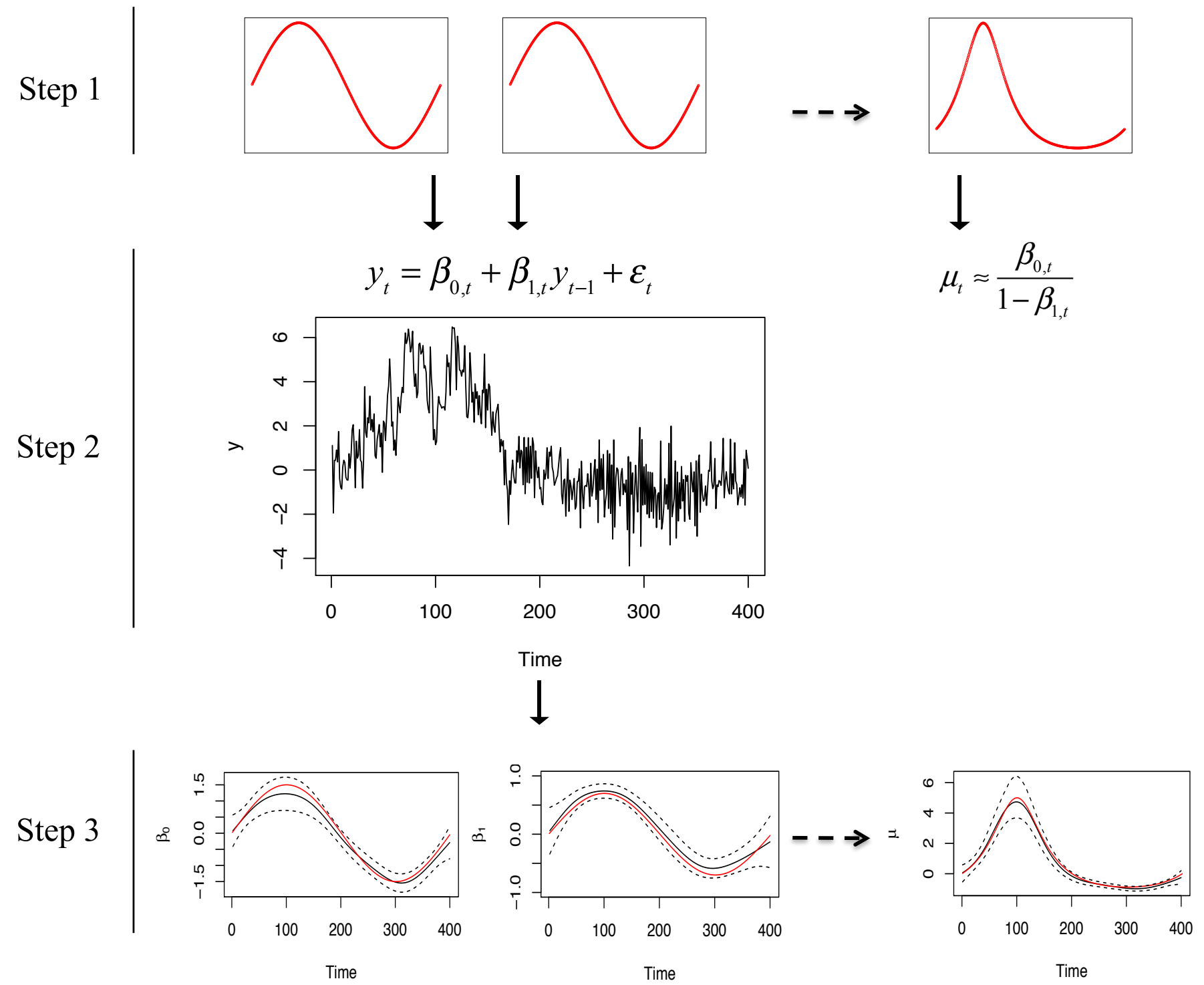

Figure 1. The simulation setup The simulation setup consists of three steps. Step 1 represents the simulation conditions, step 2 the generation of the simulation data and step 3 the estimation and evaluation of the TV-AR model. 


\section{References}

Giraitis, L., Kapetanios, G., \& Yates, T. (2014). Inference on stochastic time-varying coefficient models. Journal of Econometrics, 179(1), 46-65.

Rovine, M. J., \& Walls, T. A. (2006). Multilevel autoregressive modeling of interindividual differences in the stability of a process. In T. A. Walls \& J. L. Schafer (Eds.), Models for intensive longitudinal data (pp. 124-147). Oxford, England: Oxford University Press.

Wood, S. N. (2006). Generalized additive models: An introduction with R. Boca Raton, FL: Chapman and Hall/CRC. 